\section{Avaliação do Programa de Triagem para o Hipotiveoidismo Congênito no Estado de Sergipe}

\begin{abstract}
RESUMO
Nesta pesquisa fizemos uma avaliação da oportunidade nas diversas etapas que compõem o mecanismo de detecção do Programa de Triagem para o Hipotireoidismo Congênito, sua cobertura e a freqüência de casos no serviço público de saúde em Sergipe. Levantamos a idade de todas as crianças (2994) submetidas à triagem para detecção neonatal do hipotireoidismo congênito e fenilcetonúria em suas diversas fases, no período de junho a dezembro de 1995. A idade da criança na coleta do exame, foi de $30 \pm 19$ dias (média \pm DP). A idade, quando o resultado da triagem chegou ao centro de saúde, fol de $48 \pm 7$ dias, quando entregue à família, de $66 \pm 18$ dias e quando visto pelo pediatra da unidade, de $80 \pm 40$ dias. Crianças com resultados alterados são referidas para serviços especializados sem nenhum pacto de prioridade. Com relação à cobertura, apenas $5 \%$ das crianças do interior do Estado e $42 \%$ da capital foram examinadas. A morosidade nas etapas do programa e a baixa cobertura de crianças submetidas ao teste de triagem comprovam a hipótese de que o referido programa de governo não está atingindo seus objetivos principais que são o tratamento oportuno e a cobertura a todas as crianças recém-nascidas, a fim de que sejam prevenidas suas seqüelas. (Arq Bras Endocrinol Metab 2000;44/2: 157-61)
\end{abstract}

Unitermos: Hipotireoidismo congênito; Rastreamento neonatal; Relação custo-beneficio; Tiroxina.

\begin{abstract}
In this research we evaluated the time spent in several follow-up steps of the screening mechanism of the "Early Detection of Congenital Hypothyroidism Program", its coverage and its occurrence in the civil health service in Sergipe. To accomplish this purpose, we raised data for all the infants (2994) that had been submitted to screening for neonatal hypothyroidism and phenylketonuria in the period from June to December of 1995. We verified that the age at the time of the examination was $30 \pm 19$ days (mean $\pm S D$ ). When the result arrived at the Healthy Center was $48 \pm 7$ days, the families were informed $66 \pm 18$ days, the infants were reviewed by the Health Center pediatrician at the age of $80 \pm 40$ days. Children with altered results were still referred to specialized centers without priority. Regarding the coverage, only $5 \%$ of the infants from the interior of the state and $42 \%$ from the capital, on average, were covered by the test. This extended process and the inefficient coverage of infants submitted to screening, confirm the hypothesis that this government program is not achieving its main objectives: the timely treatment and the coverage of all newborn infants, in order to prevent their impact. (Ara Bras Endocrinol Metab 2000;44/2: 157-61)
\end{abstract}

Keywords: Congenital hypothyroidism; Screening tests; Cost-effectiveness ratio; Thyroxine.

\section{perspectivas}

\author{
Roberto J.R. Ramalho \\ Daisy P. Valido \\ Manuel H. Aguiar-Olipeira
}

\author{
Divisão de Endocrinologia, \\ Departamento de Medicina, \\ Universidade Federal de Sergipe, \\ Aracaju, SE.
}

Recebido em 31/08/1999

Revisado em 26/11/1999 e 26/01/2000 Aceito em 31/01/2000 
O HIPOTIREOIDISMO CONGÊNITO (HC) é uma causa comum de retardo mental prevenível, quando a população não dispõe de programas de detecção neonatal que propiciem o diagnóstico e o tratamento oportuno (1). No período neonatal, o quadro clínico do $\mathrm{HC}$ é, na maioria dos casos, inespecífico $(1,2)$. Na cra pré-rastrcamento para o $\mathrm{HC}$, estudos demonstraram a relação inversa entre a idade, na ocasião do início do tratamento, pontuação do teste do quociente de inteligência e relação direta com outras seqüelas neurológicas (3). Ficou comprovado que os hormônios tireoideanos exercem controle em processos interdependentes do crescimento, maturação e organização do cérebro $(4,5)$. A oportunidade perdida dos hormônios tireoideanos atuarem no tempo correto, leva progressivamente a lesões que não compensadas pela plasticidade cerebral, afetam a coordenação motora e a audição, gerando desordens no sistema vestibular, anormalidades cocleares, deficiência da orientação visual espacial, de linguagem, problemas de rendimento escolar e finalmente causando deficiência mental $\mathrm{e}$ do crescimento $(1,6,7)$. Casos tratados entre um e três meses de idade, apresentam pequeno comprometimento auditivo e pequenas disfunções motoras ou de aprendizado, apesar da grande melhora com relação ao desenvolvimento físico e mental (1,8-10).

Os programas de triagem para $\mathrm{HC}$ e fenilcetonúria foram iniciados pela Secretaria de Estado da Saúde de Sergipe (SES) em 04 de maio de 1993, utilizando um laboratório privado para a realização dos exames. O objetivo do nosso estudo foi julgar o mérito do Programa de Triagem para o Hipotireoidismo Congênito (PTHC), através da avaliação do tempo gasto nas suas diversas etapas, sua cobertura, acessibilidade e freqüência de casos no serviço público de saúde em Sergipe.

\section{MATERIAL E MÉTODOS}

Os procedimentos utilizados consistiram em medir as variáveis, residência (capital ou interior), tempo (idade) e a cobertura, nos diversos arquivos públicos, realizando percentual, média aritmética, desvio padrão (DP) e verificando a freqüência acumulada relativa (ogiva percentual) da idade das crianças testadas (11). Foram obtidos os quantitativos das triagens para o cálculo da cobertura para Aracaju e para o Estado de Sergipe, no período de 07/95 a 11/95, baseado no número de nascimento/mês e óbitos de menores de 5 dias de idade, fornecidos pela Fundação Instituto Brasileiro de Geografia e Estatística (IBGE) e pelo Sistema de Informações de Nascidos Vivos e Mortalidade da Secretaria de Estado da Saúde (SINASC/SIM/SES) para o ano de 1995. A metodologia utilizada $(12,13)$ foi baseada na fórmula: cobertura = pessoas atingidas por uma atividade x 100/população alvo. Para o cálculo específico da cobertura do PTHC, utilizamos a fórmula: cobertura global $=\mathrm{n}^{\mathrm{O}}$ de triagens $\left(\right.$ TT) $\times 100 / \mathrm{n}^{\circ}$ nascidos vivos $\mathrm{n}^{\mathrm{O}}$ de $\mathrm{RN}$ com mortalidade $<5$ dias. A cobertura, quando o número de TT foi substituído pelo teto máximo autorizado pela SES (600 exames/mês), foi denominada potencial. A cobertura foi comparada a de estados brasileiros e a da Itália que dispõem de série histórica e dados nacionais (14).

O período observado em todas as crianças testadas para a tempestividade foi de meados de junho a meados de dezembro/1995. Utilizamos como procedimento de análise, a ogiva percentual da idade das crianças testadas (15), sendo comparada a ogiva nacional dos casos de HC da Itália (14).

A apuração dos dados no local (Hospital Universitário, Centros de Saúde de referência e SES) foi manual, postcriormente por computação eletrônica, e depois realizado o seu processamento para análise (planilha eletrônica Excel 5.0).

\section{RESULTADOS}

A cobertura global do PTHC em Sergipe, segundo dados do SINASC/SIM/SES e da nossa pesquisa, foi de $15 \%$ (interior $5 \%$ e capital $42 \%$ ) e a cobertura potencial, $20 \%$ (interior $7 \%$ e capital $56 \%$ ). Com dados do IBGE e da nossa pesquisa, a cobertura potencial do Estado foi de $27 \%$.

A idade da criança na data da coleta do teste de triagem para o hipotireoidismo congênito foi $30 \pm 19$ dias ( $50 \%$ delas têm idade superior a 28 dias). Quando o resultado do teste de triagem chegou ao centro de saúde foi $48 \pm 15$ dias. A idade da criança quando o resultado do teste de triagem foi recebido pela família foi $66 \pm 24$ dias e, quando o resultado do teste de triagem foi visto pelo médico, foi de $80 \pm 40$ dias. Metade destas crianças tinham idade superior a 72 dias. $O$ tempo gasto pelo serviço de saúde entre a coleta e a entrega à família foi $39 \pm 20$ dias. O tempo gasto entre a coleta e a chegada do resultado ao centro de saúde foi $19 \pm 5$ dias e para a família buscar o exame após este ter chegado ao centro de saúde foi $18 \pm 18$ dias. O tempo gasto para a família levar o resultado do teste de triagem ao médico do serviço de saúde/SES foi $14 \pm 18$ dias. Alguns destes dados foram comparados aos dados nacionais da Itália $(14,16)$. A tabela 1 mostra a distribuição relativa da idade das crianças submetidas ao PTHC na data da coleta em Sergipe em 1995 e na Itália em 1987-90 e 1991 92. Por sua vez, a figura l compara a data na qual o 
Tabela 1 - Distribuição acumulada relativa da idade de crianças submetidas do PTHC na data da coleta em Sergipe/1995 e na Itália 1987-90/1991-92.

\begin{tabular}{cccc}
\hline $\begin{array}{c}\text { Até, idade } \\
\text { em dias }\end{array}$ & $\begin{array}{c}\text { \% Itália } \\
1987-1990\end{array}$ & $\begin{array}{c}\text { \% Itália } \\
1991-1992\end{array}$ & $\begin{array}{c}\text { \% Sergipe } \\
1995\end{array}$ \\
\hline 7 & 9 & 5 & 4 \\
14 & 42 & 50 & 17 \\
16 & 50 & 63 & 22 \\
18 & 59 & 72 & 27 \\
21 & 68 & 82 & 34 \\
28 & 85 & 93 & 49 \\
29 & 86 & 94 & 50 \\
30 & 88 & 95 & 59 \\
32 & 90 & 96 & 63 \\
35 & 92 & 96 & 70 \\
42 & 95 & 98 & 82 \\
49 & 97 & 99 & 89 \\
56 & 98 & 99 & 94 \\
63 & 99 & 99 & 99 \\
70 & 100 & 100 & 99 \\
90 & 100 & 100 & 100 \\
120 & 100 & 100 & 100 \\
\hline
\end{tabular}

Fonte: SORCINE et al., 1994 e SES-1995.

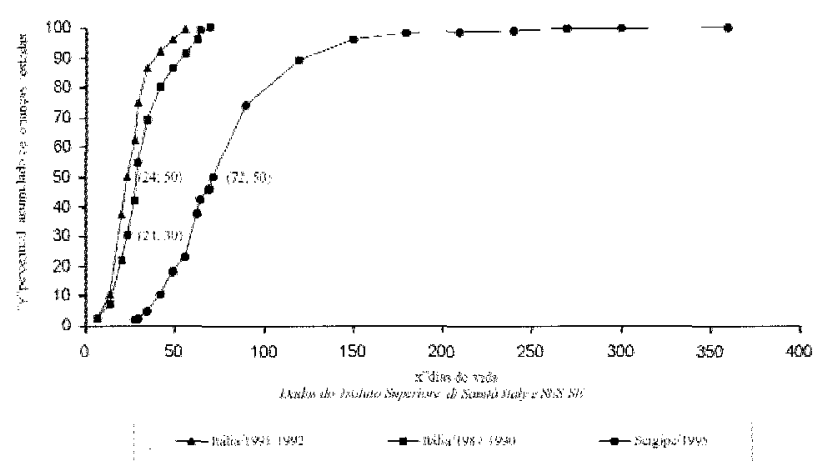

Figura 1 - Distribuição de freqüência acumulada relativa da idade de crianças submetidas ao PTHC quando o exame foi visto pelo médico em Sergipe/1995 e quando iniciou a terapia da Itália (1991-92 e 1987-90). Nos pontos estão representados os valores de $(x ; y)$.

exame foi visto pelo médico em Sergipe e quando a terapia para o $\mathrm{HC}$ foi instituída nos pacientes italianos em 1987-90 e 1991-92.

Foi diagnosticado um caso de hipotireoidismo congênito em 2994 crianças testadas. Sua coleta foi com 58 dias e o tratamento iniciado com 71 dias de idade.

\section{DISCUSSĀO}

A triagem para o $\mathrm{HC}$ apresenta sensibilidade de $97 \% \mathrm{e}$ especificidade de 99\% (17), o que a torna de valor inquestionável, sendo realizada $\mathrm{cm}$ vários países $(1,18)$. O mérito do programa, o objetivo da presente pesquisa, é a avaliação da cobertura e do tempo gasto nas diversas etapas do referido programa, visto que os atrasos no tratamento também foram provocados por problemas administrativos, principalmente nos paises tecnologicamente atrasados, comprometendo a prevenção das seqüelas do $\mathrm{HC}(1,12,17,19,20)$.

A localização geográfica dos serviços de saúde e a sua funcionalidade são fatores que interferem no acesso da população à saúde. A heterogeneidade da distribuição geográfica e das características da população conduz a refletir sobre a equiidade numa realidade concreta. Esta é entendida como igualdade de acesso, direito de todo cidadão. A acessibilidade dos serviços de saúde ć fundamental para que a eqüidade seja preservada (2l).

As baixas coberturas do PTHC em Sergipe são devidas à limitação de exames liberados pela SES, pela reduzida funcionalidade do serviço (diferença entre cobcrtura potencial e global) e pela dificuldade de acessibilidade da população do interior do Estado ( $5 \%$ e $7 \%$ coberturas global e potencial respectivamente SINASC/SIM/SES), porque o referido programa só existe na capital $(42 \%$ e $56 \%$ coberturas global c potencial respectivamente - SINASC/SIM/SES). A cobertura global de Sergipe de julho a novembro de 1995 (15\% SINASC/SIM/SES) foi comparada à da Itália (14) em 1979, sendo esta última de 97\% em 1992.

A idade da criança na data da coleta, $30 \pm 19$ dias, é extremamente tardia (17,22-31). Atualmente, o tratamento do $\mathrm{HC}$ é considerado oportuno quando iniciado até 14 dias de idade e tardio quando iniciado depois de 30 dias de vida, e deve obedecer o padrão de 3 a 5 dias para a idade na coleta do TT para o $\mathrm{HC} \mathrm{cm}$ conjunto com a da fenilcetonúria $(1,22-25,32,33)$. Crianças com $\mathrm{HC}$ não grave tratadas com $14 \pm 4$ dias na dose de $6,6 \mu \mathrm{g} / \mathrm{kg} /$ dia de tiroxina não apresentaram seqüielas (34). Pequenos danos na função neurootológica ocorrem em crianças com $\mathrm{HC}$, quando o início do tratamento foi com média de 29 dias de vida (35). Também foram verificadas alteraçōes neurofisiológicas, apesar do desenvolvimento mental normal, em crianças com início do tratamento em média de 33 dias de vida (10). Crianças com $\mathrm{HC}$ grave tratadas com 14 dias de vida na dose de $11,6 \mu \mathrm{g} / \mathrm{kg} /$ dia, não apresentaram conseqüências da enfermidade (36). O tempo gasto entre a coleta e a chegada do resultado ao centro de saúde foi de $19 \pm 5$ dias, muito maior que o recomendado de 3 dias úteis (26), conduzindo a um atraso adicional. Na Itália (14,16), em 1991-1992, $50 \%$ das crianças tinham idade até 14 dias na coleta $\mathrm{c}$ 24 dias no início do tratamento, antecipando a coleta (16 dias) e a terapia (29 dias) do período 1987-1990. 
Em Sergipe estes prazos são demasiadamente retardados, $50 \%$ das crianças têm 29 dias de idade na coleta e 72 dias quando o resultado foi visto pelo médico. No único caso de $\mathrm{HC}$ diagnosticado em Sergipe pelo PTHC, no período analisado, o tratamento foi iniciado muito tardiamente com 71 dias de idade. Hoje o PTHC oportuno e universal, além de evitar o retardo mental grave, também previne os pequenos danos ao Sistema Nervoso Central (SNC).

As variáveis tempo da coleta até a entrega do resultado e cobertura do P'THC também exprimem a acessibilidade, disponibilidade e relevância dos atores sociais envolvidos no exame. A dificuldade de acessibilidade manifesta-se, principalmente, no usuário do interior do Estado de Sergipe, visto que sua procura pelos serviços é diminuta, por ser a ação executada apenas na capital. A precária oportunidade e a baixa cobertura reduzem sobremaneira o mérito do referido programa e representam a interação de poderes, interesses e necessidades envolvidos, expressando um comportamento social frente a uma política governamental. O Brasil é hoje um país que está entre as primeiras economias do mundo, apesar dos baixos índices de desenvolvimento humano (37). Isso faz com que surjam problemas de saúde como as doenças crônicas degenerativas e do metabolismo (38), aliadas às doenças da miséria $(39,40)$. Os recursos que a nossa economia proporciona podem amenizar estes problemas através da sua redistribuição. As doenças da miséria são resolvidas com modificações na estrutura social, o que inclui a melhora do nível de vida da população. Portanto, é pertinente investir naquilo que é específico para saúde, como a prevenção das seqüelas do $\mathrm{HC}$, havendo necessidade de ampliar a cobertura com um P'THC oportuno a todos RN de Sergipe.

A presente pesquisa foi a primeira dissertação de mestrado da área de saúde na UFS. Utilizando metodologia de baixo custo, provocou um impacto político de grandes resultados. Dois novos laboratórios foram montados, um no Laboratório Central da SES e outro no Hospital Universitário para também realizarem o PTHC. Uma coordenação única a cargo do principal autor deste trabalho, trabalha com o objetivo de melhorar a dramática situação atual. Uma permanente sinergia entre as diversas instituições envolvidas é necessária para a consecução deste objetivo de grande impacto social.

\section{AGRADECIMENTOS}

À Secretaria de Estado da Saúde de Sergipe e ao Núcleo de Pós-Graduação em Medicina da Universidade Federal de Sergipe.

\section{REFERÊNCIAS}

1. LaFranchi S. Detecção neonatal de hipotiroidismo congênito: Uma história de sucesso? Arq Bras Endocrinol Metab 1995:39:80-8.

2. Damiani $D$, Forte $W C N$, Dichtchekenian $V$, Deluqui $C G$. Hipotireoidismo congênito: evolução de crianças tratadas. J Pediatr (Rio J) 1981:51:187-90.

3. Klein AH, Meltzer S, Kenny FM. Improved prognosis in congenital hypothyroidism treated before age three months. J Pediatr 1972:81:912-5.

4. Escobar GM de, Escobar del Rey F, Obregon MJ, RuizMarcos A. The hypothyroid rat. In : Medeiros-Neto, et al. lodine Deficiency Disorders and Congenital Hypothyroidism. São Paulo: Univ. São Paulo (USP), 1986: 52-64.

5. Ford DH, Cramer EB. Developing Nervous System in Relation to Thyroid Hormones. In: Grave GD, ed. Thyroid Hormones and Brain Development. New York: Congress; Raven Press, 1977: 1-18.

6. O'Malley Jr BW, LI D, Turner DS. Hearing loss and cochlear abnormalities in the congenital hypothyroid (hyp/hyt) mouse. Hearing Research 1995:88:181-9.

7. Rybak LP. Metabolic disorders of the vestibular system. Otolaryngol Head Neck Surg 1995; 1 12(1):128-32.

8. Botler J. Repercussões neurológicas do hipotireoidismo congênito. Arq Bras Endocrinol Metab 1996;40(4): 264-70.

9. Glorieux J, Dussault JH, Morissette J, Desjardins J, Letarte J. Guyda H. Follow-up of ages 5 and 7 years on mental development in children with hypothyroidism detected by Quebec screening program. J Pediatr 1986; 107:913-5.

10. Weber $G$, Siragusa $V$, Rondanini GF, Prina Cerai $L M$, Mora S, Colmbini J et al. Neurophysiologic studies and cognitive function in congenital hypothyroid Children. Pediatric Res 1995;37(6):736-40.

11. Berquó ES, Souza JMP, Gotlieb SLD. Bioestatística. São Paulo: EPU, 1981;11-160.

12. Aguilar MJ, Ander-Egg E. Avaliação de Serviços e Programas Sociais. Petrópolis: Vozes, 1994:9-149.

13. Rouquayrol MZ, Fischmann A, Santos AN. Epidemiologia \& Saúde. $2^{a}$, ed. Rio de Janeiro: Medsi, 1986; 13-237.

14. Sorcini M, Fazzini C, Oliveiri A, Grandolfo ME, Medda E, Stazi MA et al. Lo screening neonatale dell ipotiroidismo congenito in Italia. II registro nazionale. Ann Ist Super Sanità 1994;30:275-87.

15. Blanco-Lopez A, Amarilla T, Dorantes-Alvarez LM. Evaluación clínica y de laboratorio en pacientes con hipotihoidismo congénito. Bol Med Hosp Infant Mex 1986:43:228-32.

16. Cassio A, Piazzi S, Colli C, Balsamo A, Bozza D, Salardi S, et al. Modelli di organizzazione degli screening neonatali. Ann Ist Super Sanità 1994:30:269-73.

17. Pharoah POD, Madden MP. Audit of screening for hypothyroidism. Arch Dis Child 1992;67: 1073-6. 
18. Toublanc JE. Comparison of epidemiological data on congenital hypothyroidism in Europe with those of other parts in the world. Horm Res 1992;38:230-5.

19. Fisher DA. Effectiveness of newborn screening program for congenital hypothyroidism: prevalence of missed. Ped Clin N Am 1987:34:881-90.

20. Dussault JH, Mitchell ML, LaFranchi S, Murphey WH Regional screening for Congenital Hypothyroidism: results of screening one million North American infants with filter paper spot T4-TSH. In: Burrow GN, Dussualt JH, eds. Neonatal Thyroid Screening. New York: Raven Press, 1980: 155-65.

21. Unglert CV de S. O enfoque da acessibilidade no planejamento da localização e dimensão de serviços de saúde. Rev Saúde Públ 1990; São Paulo, 24(6):445-52.

22. Alm J, Hagenfeldt L, Larsson A, Lundberg K. Incidence of congenital hypothyroidism: retrospective study of neonatal laboratory screening versus clinical symptoms as indicators leading to diagnosis. $\mathrm{Br}$ Med $\mathrm{J}$ 1984;2893: $1171-4$

23. Fisher DA. Clinical Review 19. Management of Congenital Hypothyroidism. J Clin Endocrinol Metab 1991; 72:523-9.

24. Rovet JF, Ehrlich RM. Long-term effects of L-thyroxin Therapy for congenital hypothyroidism. J Pediatr $1995 ; 126: 380-6$

25. Zetterström R. Editorial comment on phenylketonuria. Acta Paediatric 1995:84:716-8.

26. Majeed-Saidan MA, Joyce B, Khan M, Hamam HD. Congenital hypothyroidism: the Riyadh Military Hospital experience. Clin Endocrinol 1993:38:191-5.

27. Burgard P, Armbruster M. Schmidt E, Rupp A. Psychopathology of patients treated early for phenylketonuria: results of the German collaborative study of phenylketonuria. Acta Paediatr 1994;407: 108-10.

28. Frank JE, Faix JE, Hermos RJ, Mullaney DM, Rojan DA Mitchell ML, ef al. Thyroid function in very low birth weight infants: effects on neonatal hypothyroidism screening. J Pediatr 1996; 128:548-54.

29. Irons M. Screening for metabolical disorders. Pediatr Clin North Am 1993:40:1073-85.

30. Saslow JG, Post EM, Southard CA. Thyroid screening for early discharged infants. Pediatrics 1996;98:41-3.
31. Verkerk PH, Buitendijk SE, Verloove-Vanhorick P. Congenital hypothyroidism screening and the cutoff for thyrotropin measurement: recommendations from the Netherlands. Am J Public Health 1993;83:868-71.

32. Glorieux J, Desjardins M, Letarte J, Morissette J, Dussault $\mathrm{JH}$. Useful parameters to predict the eventual mental outcome of hypothyroid children. Pediatric Res $1988 ; 24: 6-8$

33. Walfish PG, Cord VS. Heel blood screening for neonatal hypothyroidism. In: Medeiros Neto $G$, Maciel R, eds. lodine Deficiency Disorders and Congenital Hypothyroidism. São Paulo; Aché:1986; 194-202

34. Campos SP, Sandberg DE, Barrick C, Voorhess ML, Macgillivray $\mathrm{MH}$. Outcome of lower l-thyroxine dose for treatment of congenital hypothyroidism. Clin Pediatr $1995: 34: 514-20$

35. Bellman SC, Davies A, Fuggle PG, Grant DB, Smith I. Mild impairment of neuro-otological function in early treated congenital hypothyroidism. Arch Dis Child 1996;74:215-8.

36. Dubuis JM, Glorieux J, Richer F, Deal CL, Dussault JH, Vliet GV. Outcome of severe congenital hypothyroidism: closing the developmental gap with early high dose levothyroxine treatment. J Clin Endocrinol Metab $1996 ; 81: 222-7$

37. UNICEF. Situação Mundial da Infância. Brasillia $1994 ; 62-87$

38. Sounis E. Epidemiologia. Rio de Janeiro. São Paulo: Atheneu; 1985:2:295-8.

39. Fundação Nacional DE Saúde. Manual da Dengue, vigilância epidemiológica e atençāo ao doente. Brasilia 1996;7.

40. Singer $P$, Campos O, Oliveira EM. Prevenir e curar: o controle social atravès dos serviços de saúde. Rio de Janeiro: Forense, 1981:117-21.

\section{Endereço para correspondência:}

Manuel H. Agular-Oliveira

Departamento de Medicina - Hospital Universitário

Rua Cláudio Batista s/n - Sanatório

49060-100 Aracaju, Sergipe

Fax: (0XX79) 214-1039 